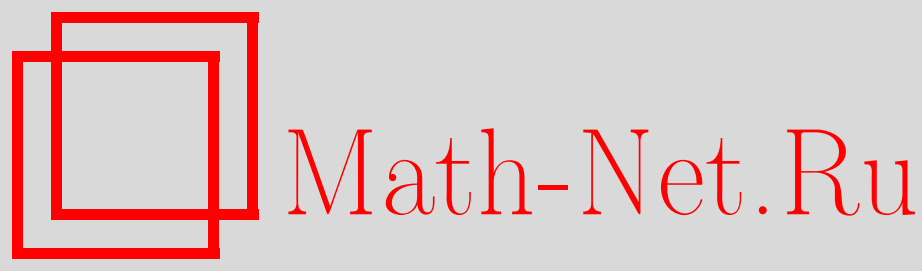

В. Е. Тарасов, Дробные интегро-дифференциальные уравнения для электромагнитных волн в диэлектрических средах, ТМФ, 2009, том 158, номер 3, 419-424

DOI: https://doi.org/10.4213/tmf6324

Использование Общероссийского математического портала Math-Net.Ru подразумевает, что вы прочитали и согласны с пользовательским соглашением http://www . mathnet.ru/rus/agreement

Параметры загрузки:

IP : 54.196 .121 .252

26 апреля 2023 г., 04:41:07

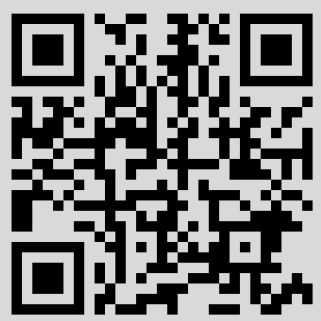




\title{
ФИЗИКА
}

Том 158, № 3

март, 2009

2009 г.

B. E. Tapacoв*

\section{ДРОБНЫЕ ИНТЕГРО-ДИФФЕРЕНЦИАЛЬНЫЕ УРАВНЕНИЯ ДЛЯ ЭЛЕКТРОМАГНИТНЫХ ВОЛН В ДИЭЛЕКТРИЧЕСКИХ СРЕДАХ}

\begin{abstract}
Доказано, что электромагнитные поля в диэлектрических средах, восприимчивость которых в широком частотном диапазоне подчиняется дробно-степенной зависимости, описываются дифференциальными уравнениями с производными нецелого порядка по времени. Получены дробные интегро-дифференциальные уравнения для электромагнитных волн в диэлектрике. Электромагнитные поля в диэлектриках демонстрируют дробно-степенную релаксацию. Дробные интегро-дифференциальные уравнения для электромагнитных волн являются общими для широкого класса диэлектрических сред независимо от физической структуры, химического состава или природы поляризации (дипольная, электронная или ионная).
\end{abstract}

Ключевые слова: дробное интегро-дифференцирование, дробное затухание, универсальный ответ, электромагнитное поле, диэлектрические среды.

\section{1. ВВЕДЕНИЕ}

В 1912 г. Дебай сформулировал теорию дипольной релаксации в диэлектриках [1]. Большое количество проведенных измерений диэлектрической релаксации показывает, что классическое дебаевское поведение почти никогда не наблюдается экспериментально [2]-[4]. Фактически оказывается, что различные диэлектрики демонстрируют степенные закономерности, подтверждая диэлектрические измерения Джошера [2], [3] для широкого класса различных веществ.

Для большинства материалов диэлектрическая восприимчивость в широкой частотной области подчиняется дробно-степенному закону, называемому универсальным ответом [2], [3]. Эта закономерность была обнаружена в биполярных средах вне области частот пиковой потери и в средах, где поляризация является результатом движений ионных или электронных носителей зарядов. Было показано [5], что частотная зависимость диэлектрической восприимчивости $\widetilde{\chi}(\omega)=\chi^{\prime}(\omega)-i \chi^{\prime \prime}(\omega)$

${ }^{*}$ Научно-исследовательский институт ядерной физики им. Д. В. Скобельцына, Московский государственный университет им. М. В. Ломоносова, Москва, Россия.

E-mail: tarasov@theory.sinp.msu.ru 
подчиняется общим универсальным законам для широкого класса сред. А именно, закономерности, описываемые соотношениями

$$
\begin{array}{lll}
\chi^{\prime}(\omega) \sim \omega^{n-1}, & \chi^{\prime \prime}(\omega) \sim \omega^{n-1}, & \omega \gg \omega_{p}, \\
\chi^{\prime}(0)-\chi^{\prime}(\omega) \sim \omega^{m}, & \chi^{\prime \prime}(\omega) \sim \omega^{m}, & \omega \ll \omega_{p},
\end{array}
$$

где $\chi^{\prime}(0)$ - статическая поляризация, $0<n, m<1, \omega_{p}$ - частота пика потерь, наблюдаются в широком диапазоне частот. Отметим, что отношение мнимой и действительной компонент восприимчивости не зависит от частоты. Частотная зависимость, заданная соотношением (1), подразумевает, что мнимая и действительная части комплексной восприимчивости при больших частотах подчиняются соотношению

$$
\frac{\chi^{\prime \prime}(\omega)}{\chi^{\prime}(\omega)}=\operatorname{cth} \frac{\pi n}{2}, \quad \omega \gg \omega_{p} .
$$

Экспериментальная зависимость (2) приводит к похожему правилу частотной независимости для низкочастотной области:

$$
\frac{\chi^{\prime \prime}(\omega)}{\chi^{\prime}(0)-\chi^{\prime}(\omega)}=\operatorname{th} \frac{\pi m}{2}, \quad \omega \ll \omega_{p} .
$$

Законы универсального ответа диэлектрических сред [2], [3] могут описываться методами дробного математического анализа [6]. Теория интегралов и производных нецелого порядка восходит к работам Лейбница, Лиувилля, Римана, Грюнвальда и Летникова [6]. Дробный математический анализ находит множество применений в современных исследованиях в механике и физике. Интерес к дробным интегро-дифференциальным уравнениям непрерывно растет в последние годы в силу их многочисленных приложений. В короткий период времени список применений стал очень большим (см., например, [7]-[9]). Отметим работы [10]-[13], в которых дробный математический анализ был применен для объяснения природы неэкспоненциальных релаксаций и были получены уравнения, содержащие дробное интегрирование и дифференцирование.

В настоящей работе показано, что дробно-степенная частотная зависимость приводит к интегро-дифференциальным уравнениям с производными и интегралами нецелого порядка по времени. Выводятся дробные дифференциальные уравнения, которые описывают электромагнитные волны в широком классе диэлектрических сред. Степенные законы Джошера представляются дробными интегро-дифференциальными уравнениями. Электромагнитные поля в диэлектрических средах демонстрируют универсальное затухание с дробными показателями. Предлагаемые дробные уравнения являются общими (универсальными) для широкого класса материалов независимо от их физической структуры, химического состава или природы их поляризации.

\section{2. ДРОБНЫЕ УРАВНЕНИЯ ДЛЯ УНИВЕРСАЛЬНЫХ ЗАКОНОВ}

Рассмотрим уравнения (1) и (3). Для области $\omega \gg \omega_{p}$ универсальный дробно-степенной закон (1) может быть представлен в виде

$$
\tilde{\chi}(\omega)=\chi_{\alpha}(i \omega)^{-\alpha}, \quad 0<\alpha<1,
$$


с некоторыми положительными постоянными $\chi_{\alpha}$ и $\alpha=1-n$, где

$$
(i \omega)^{\alpha}=|\omega|^{\alpha} e^{i \alpha \pi \operatorname{sgn}(\omega) / 2} .
$$

Легко увидеть, что соотношение (3) справедливо для закона (5).

Плотность поляризации $\mathbf{P}(t, r)$ может быть записана в виде

$$
\mathbf{P}(t, r)=\mathcal{F}^{-1}(\widetilde{\mathbf{P}}(\omega, r))=\varepsilon_{0} \mathcal{F}^{-1}(\widetilde{\chi}(\omega) \widetilde{\mathbf{E}}(\omega, r)),
$$

где $\widetilde{\mathbf{P}}(\omega, r)$ - преобразование Фурье $\mathcal{F}$ функции $\mathbf{P}(t, r)$. При подстановке соотношения (5) в уравнение (6) получаем

$$
\mathbf{P}(t, r)=\varepsilon_{0} \chi_{\alpha} \mathcal{F}^{-1}\left((i \omega)^{-\alpha} \widetilde{\mathbf{E}}(\omega, r)\right) .
$$

Заметим, что преобразование Фурье дробного интеграла Лиувилля [6], [14]

$$
\left(I_{+}^{\alpha} f\right)(t)=\frac{1}{\Gamma(\alpha)} \int_{-\infty}^{t} \frac{f\left(t^{\prime}\right) d t^{\prime}}{\left(t-t^{\prime}\right)^{1-\alpha}}
$$

задается следующим соотношением (см. [6], теорема 7.1 и [14], теорема 2.15):

$$
\left(\mathcal{F} I_{+}^{\alpha} f\right)(\omega)=\frac{1}{(i \omega)^{\alpha}}(\mathcal{F} f)(\omega)
$$

где $0<\operatorname{Re} \alpha<1$ и $f(t) \in L_{1}(\mathbb{R})$ или $1 \leqslant p<1 / \operatorname{Re} \alpha$ и $f(t) \in L_{p}(\mathbb{R})$.

Используя дробный интеграл Лиувилля и дробно-степенной закон $(5)$ для $\widetilde{\chi}(\omega)$ в частотной области, получаем

$$
\mathbf{P}(t, r)=\varepsilon_{0} \chi_{\alpha}\left(I_{+}^{\alpha} \mathbf{E}\right)(t, r), \quad 0<\alpha<1 .
$$

Это уравнение показывает, что плотность поляризации $\mathbf{P}(t, r)$ для области высоких частот пропорциональна дробному интегралу Лиувилля от электрического поля $\mathbf{E}(t, r)$.

Рассмотрим уравнения (2) и (4). Для области частот $\omega \ll \omega_{p}$ универсальный дробно-степенной закон (2) может быть представлен в виде

$$
\tilde{\chi}(\omega)=\tilde{\chi}(0)-\chi_{\beta}(i \omega)^{\beta}, \quad 0<\beta<1,
$$

с некоторыми положительными константами $\chi_{\beta}, \widetilde{\chi}(0), \beta=m$. Несложно доказать, что для (8) справедливо равенство (4).

Отметим, что преобразование Фурье дробной производной Лиувилля

$$
\left(D_{+}^{\beta} f\right)(t)=\frac{\partial^{k}}{\partial t^{k}}\left(I_{+}^{k-\beta} f\right)(t)=\frac{1}{\Gamma(k-\beta)} \frac{\partial^{k}}{\partial t^{k}} \int_{-\infty}^{t} \frac{f\left(t^{\prime}\right) d t^{\prime}}{\left(t-t^{\prime}\right)^{\beta-k+1}},
$$

где $k-1<\beta<k$, задается следующей формулой (см. [6], теорема 7.1 и [14], теорема 2.15):

$$
\left(\mathcal{F} D_{+}^{\beta} f\right)(\omega)=(i \omega)^{\beta}(\mathcal{F} f)(\omega),
$$

где $0<\operatorname{Re} \beta<1$ и $f(t) \in L_{1}(\mathbb{R})$ или $1 \leqslant p<1 / \operatorname{Re} \beta$ и $f(t) \in L_{p}(\mathbb{R})$. 
Используя определение дробной производной Лиувилля и дробно-степенной закон (8), плотность поляризации (6) можно задать в виде

$$
\mathbf{P}(t, r)=\varepsilon_{0} \tilde{\chi}(0) \mathbf{E}(t, r)-\varepsilon_{0} \chi_{\beta}\left(D_{+}^{\beta} \mathbf{E}\right)(t, r), \quad 0<\beta<1 .
$$

Это соотношение показывает, как плотность поляризации $\mathbf{P}(t, r)$ в области низких частот определяется дробной производной Лиувилля от электрического поля $\mathbf{E}(t, r)$.

Соотношения (7) и (9) могут рассматриваться как уравнения универсальных законов. Эти уравнения, содержащие интегрирования и дифференцирования нецелых порядков, позволяют получить дробные волновые уравнения для электрического и магнитного полей.

\section{3. УРАВНЕНИЕ УНИВЕРСАЛЬНЫХ ЭЛЕКТРОМАГНИТНЫХ ВОЛН}

В этом разделе мы получим дробное дифференциальное уравнение для электромагнитных полей в диэлектрических средах. Используя уравнения Максвелла, имеem

$$
\varepsilon_{0} \frac{\partial^{2} \mathbf{E}(t, r)}{\partial t^{2}}+\frac{\partial^{2} \mathbf{P}(t, r)}{\partial t^{2}}+\frac{1}{\mu}\left(\operatorname{grad} \operatorname{div} \mathbf{E}-\nabla^{2} \mathbf{E}\right)+\frac{\partial \mathbf{j}(t, r)}{\partial t}=0 .
$$

При $\omega \gg \omega_{p}$ плотность поляризации $\mathbf{P}(t, r)$ связана с полем $\mathbf{E}(t, r)$ с помощью уравнения (7). Подставляя соотношение (7) в уравнение (10), получаем дробное дифференциальное уравнение для напряженности электрического поля

$$
\frac{1}{v^{2}} \frac{\partial^{2} \mathbf{E}(t, r)}{\partial t^{2}}+\frac{\chi_{\alpha}}{v^{2}}\left(D_{+}^{2-\alpha} \mathbf{E}\right)(t, r)+\left(\operatorname{grad} \operatorname{div} \mathbf{E}-\nabla^{2} \mathbf{E}\right)=-\mu \frac{\partial \mathbf{j}(t, r)}{\partial t},
$$

где $0<\alpha<1, v^{2}=1 /\left(\varepsilon_{0} \mu\right)$. Заметим, что $\operatorname{div} \mathbf{E} \neq 0$ для $\rho(t, r)=0$.

В области частот $\omega \ll \omega_{p}$ функции $\mathbf{P}(t, r)$ и $\mathbf{E}(t, r)$ связаны соотношением (9). В этом случае уравнение (10) принимает вид

$$
\frac{1}{v_{\beta}^{2}} \frac{\partial^{2} \mathbf{E}}{\partial t^{2}}-\frac{a_{\beta}}{v_{\beta}^{2}}\left(D_{+}^{2+\beta} \mathbf{E}\right)+\left(\operatorname{grad} \operatorname{div} \mathbf{E}-\nabla^{2} \mathbf{E}\right)=-\mu \frac{\partial \mathbf{j}}{\partial t}, \quad 0<\beta<1,
$$

где

$$
v_{\beta}^{2}=\frac{1}{\varepsilon_{0} \mu[1+\widetilde{\chi}(0)]}, \quad a_{\beta}=\frac{\chi_{\beta}}{1+\widetilde{\chi}(0)} .
$$

Уравнения (11), (12) описывают изменение напряженности электрического поля в диэлектрических средах. Они являются дробными дифференциальными уравнениями [14], содержащими производные порядка $2-\alpha$ и $2+\beta$.

Используя уравнения Максвелла, получаем уравнение для индукции магнитного поля

$$
\frac{\partial^{2} \mathbf{B}(t, r)}{\partial t^{2}}=\frac{1}{\varepsilon_{0} \mu} \nabla^{2} \mathbf{B}(t, r)+\frac{1}{\varepsilon_{0}} \frac{\partial}{\partial t} \operatorname{rot} \mathbf{P}(t, r)+\frac{1}{\varepsilon_{0}} \operatorname{rot} \mathbf{j}(t, r) .
$$

В экспериментах поле $\mathbf{B}(t, r)$ может реализовываться как $\mathbf{B}(t, r)=0$ для $t \leqslant 0$ и $\mathbf{B}(t, r) \neq 0$ для $t>0$. При $\omega \gg \omega_{p}$ плотность поляризации $\mathbf{P}(t, r)$ связана с полем $\mathbf{E}(t, r)$ с помощью уравнения (7), что приводит к дробному дифференциальному 
уравнению для индукции магнитного поля вида

$$
\frac{1}{v^{2}} \frac{\partial^{2} \mathbf{B}(t, r)}{\partial t^{2}}+\frac{\chi_{\alpha}}{v^{2}}\left({ }_{0} D_{t}^{2-\alpha} \mathbf{B}\right)(t, r)-\nabla^{2} \mathbf{B}(t, r)=\mu \operatorname{rot} \mathbf{j}(t, r)
$$

где $0<\alpha<1, v^{2}=1 /\left(\varepsilon_{0} \mu\right),{ }_{0} D_{t}^{2-\alpha}$ - дробная производная Римана-Лиувилля [14] на полуоси $[0, \infty)$ такая, что

$$
\left({ }_{0} D_{t}^{2-\alpha} f\right)(t)=\frac{1}{\Gamma(\alpha)} \frac{\partial^{2}}{\partial t^{2}} \int_{0}^{t} \frac{f\left(t^{\prime}\right) d t^{\prime}}{\left(t-t^{\prime}\right)^{1-\alpha}}, \quad 0<\alpha<1 .
$$

При $\omega \ll \omega_{p}$ получаем уравнение

$$
\frac{1}{v_{\beta}^{2}} \frac{\partial^{2} \mathbf{B}(t, r)}{\partial t^{2}}-\frac{a_{\beta}}{v_{\beta}^{2}}\left({ }_{0} D_{t}^{2+\beta} \mathbf{B}\right)(t, r)-\nabla^{2} \mathbf{B}(t, r)=\mu \operatorname{rot} \mathbf{j}(t, r),
$$

где $0<\beta<1$ и

$$
v_{\beta}^{2}=\frac{1}{\varepsilon_{0} \mu[1+\tilde{\chi}(0)]}, \quad a_{\beta}=\frac{\chi_{\beta}}{1+\tilde{\chi}(0)} .
$$

Уравнения (14), (15) являются дробными дифференциальными уравнениями, которые описывают магнитное поле в диэлектрических средах, демонстрирующих степенной закон релаксации. Они могут быть записаны в обобщенной форме. Такое общее дробное дифференциальное уравнение для индукции магнитного поля имеет вид

$$
\left({ }_{0} D_{t}^{\alpha} \mathbf{B}\right)(t, r)-\lambda_{1}\left({ }_{0} D_{t}^{\beta} \mathbf{B}\right)(t, r)-\lambda_{2} \nabla^{2} \mathbf{B}(t, r)=\mathbf{f}(t, r),
$$

где $1 \leqslant \beta<\alpha<3$. Здесь ротор плотности электрического тока свободных зарядов рассматривается как внешний источник: $\mathbf{f}(t, r)=\mu \lambda_{2} \operatorname{rot} \mathbf{j}(t, r)$. Уравнение (16) дает соотношение (14) для $\alpha=2,1<\beta<2$ и $\lambda_{1}=-\chi_{\alpha}, \lambda_{2}=v^{2}=1 /\left(\varepsilon_{0} \mu\right)$. Уравнение (15) можно записать в форме (16) при $2<\alpha<3, \beta=2$ и

$$
\lambda_{1}=\frac{1}{a_{\beta}}=\frac{1+\tilde{\chi}(0)}{\chi_{\beta}}, \quad \lambda_{2}=-\frac{v_{\beta}^{2}}{a_{\beta}}=\frac{-1}{\varepsilon_{0} \mu \chi_{\beta}} .
$$

Точное решение уравнения (16) может быть записано в терминах функции Райта (см. [14], теорема 5.5). Заметим, что функции Райта могут быть представлены как производные от функции Миттаг-Леффлёра $E_{\alpha, \beta}[z]$ (см. [14]). Решения уравнения (16) описывают дробно-степенное затухание магнитного поля в диэлектрических средах. Важным свойством эволюции, описываемой дробным дифференциальным уравнением, является существование дробно-степенных асимптотик для их решений.

\section{4. ЗАКЛЮЧЕНИЕ}

В настоящей работе показано, что электромагнитные поля и волны для широкого класса диэлектрических сред описываются дробными дифференциальными уравнениями с производными по времени порядка $2-\alpha$ и $2+\beta$, где $0<\alpha<1$ и $0<\beta<1$. Параметры $\alpha=1-n$ и $\beta=m$ определяются показателями $n$ и $m$, фигурирующими в экспериментально измеримых частотных зависимостях диэлектрической восприимчивости, называемых законами универсального ответа. Важным свойством 
динамики электромагнитного поля в диэлектриках, описываемого дробными дифференциальными уравнениями, является существование дробно-степенных хвостов у решений этих уравнений. Предлагаемые дробные дифференциальные уравнения для универсальных электромагнитных волн в диэлектрике являются общими (универсальными) для широкого класса сред независимо от их физической структуры, химического состава и природы поляризации (дипольная, электронная или ионная).

Отметим, что дифференциальные уравнения с производными нецелого порядка, предложенные для описания электромагнитного поля в диэлектрических средах, могут решаться численно. Например, схема дискретизации Грюнвальда-Летникова [6] применяется для численного моделирования электромагнитного поля в диэлектриках, описываемых дробными дифференциальными уравнениями. При малых отклонениях величин $\alpha$ или $\beta$ от целых значений можно использовать $\varepsilon$-разложение [15] по малому параметру $\varepsilon=\alpha$ или $\varepsilon=1-\beta$. Следует отметить, что физическая интерпретация дробных интегралов и производных может быть связана с эффектами памяти или фрактальными свойствами сред (см., например, [16], [17]).

\section{Список литературы}

[1] P. Debye, Physik. Zs., 13 (1912), 97-100.

[2] A. K. Jonscher, Universal Relaxation Law, Chelsea Dielectrics Press, London, 1996.

[3] A. K. Jonscher, J. Physics D, 32:14 (1999), R57-R70.

[4] T. V. Ramakrishnan, M. R. Lakshmi (eds.), Non-Debye Relaxation in Condensed Matter, World Scientific, Singapore, 1987.

[5] A. K. Jonscher, Nature, 267:5613 (1977), 673-679; Philos. Magazine B, 38:6 (1978), 587-601.

[6] С. Г. Самко, А. А. Килбас, О. И. Марычев, Интегралы и производные дробного порядка и некоторые их приложения, Наука и техника, Минск, 1987.

[7] G. M. Zaslavsky, Hamiltonian Chaos and Fractional Dynamics, Oxford Univ. Press, Oxsford, 2005.

[8] A. Carpinteri, F. Mainardi, Fractals and Fractional Calculus in Continuum Mechanics, CISM Courses and Lectures, 378, Springer, Wien, 1997.

[9] R. Hilfer (ed.), Applications of Fractional Calculus in Physics, World Scientific, Singapore, 2000.

[10] Р. Р. Нигматуллин, Я. Е. Рябов, ФТT, 39:1 (1997), 101-105.

[11] V. V. Novikov, V.P. Privalko, Phys. Rev. E, 64:3 (2001), 031504.

[12] Y. Yilmaz, A. Gelir, F. Salehli, R. R. Nigmatullin, A. A. Arbuzov, J. Chem. Phys., 125:23 (2006), 234705.

[13] R. R. Nigmatullin, A. A. Arbuzov, F. Salehli, A. Giz, I. Bayrak, H. Catalgil-Giz, Physica B, 388:1-2 (2007), 418-434.

[14] A. A. Kilbas, H. M. Srivastava, J. J. Trujillo, Theory and Application of Fractional Differential Equations, North-Holland Math. Stud., 204, Elsevier, Amsterdam, 2006.

[15] V. E. Tarasov, G. M. Zaslavsky, Physica A, 368:2 (2006), 399-415.

[16] Р. Р. Нигматуллин, ТМФ, 90:3 (1992), 354-368.

[17] А. А. Станиславский, ТМФ, 138:3 (2004), 491-507.

Поступила в редакцию 18.12.2007, после доработки 24.06.2008 